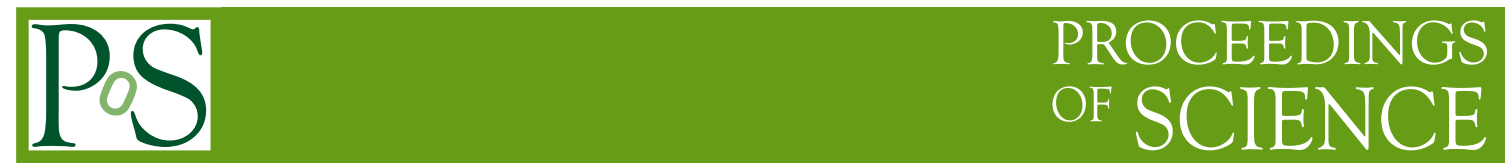

\title{
On the structure of QCD confining string
}

\author{
P.Yu. Boyko, F.V. Gubarev*, S.M. Morozov, \\ ITEP, B.Cheremushkinskaya 25, Moscow, 117218, Russia
}

\begin{abstract}
The action density distribution associated with confining flux tube is investigated in SU(2) lattice gauge theory at widely varied lattice spacing. It is argued that the gluon condensate vanishes on the string symmetry axis. We qualitatively confirm the widening of the string with increase of sources separation, however, the broadening seems to be a subleading effect. Instead, it is argued that the string width shrinks linearly with lattice spacing and the action density diverges quadratically keeping the static potential cutoff independent.
\end{abstract}

The XXV International Symposium on Lattice Field Theory

July 30 - August 42007

Regensburg, Germany

${ }^{*}$ Speaker. 
It is commonly believed that quark confinement happens due to formation of thick chromoelectric string spanned between color sources, which due to its non-zero tension confines quarks into colorless hadrons. However, apart from the non-zero physical tension the model independent properties of QCD string are still mostly unknown. In this respect the lattice simulations are indispensable, see, e.g., Refs. [1,2] and references therein. In particular, the QCD string manifests itself in the suppression of the gluonic action density, which is directly observable in numerical experiments.

In this paper we report (slightly updated with respect to [3], see below) measurements of the action density distribution associated with the confining flux tube in quenched SU(2) lattice gauge theory. Our geometrical setup is as follows. The static quark-antiquark pair created at the Euclidean time $t=0$ and annihilated at $t=T$ is represented by rectangular $T \times R$ Wilson loop. The elapsed time $T$ is asserted to be large enough to observe fully developed string at $t=T / 2$, where the numerical evaluation of the string action density profile is performed. We consider the correlation function

$$
\Delta s(x, T, R)=\langle s\rangle_{0}-\langle s\rangle_{W}=\langle s\rangle_{0}-\frac{\langle s(x) W(T, R)\rangle}{\langle W(T, R)\rangle},
$$

which is the difference of the action density $s=1 / 2 \operatorname{Tr} F_{\mu v}^{2}$ in the vacuum, $\langle s\rangle_{0}$, and its expectation value evaluated in the presence of heavy quark-antiquark pair at spatial coordinates $x$ from the Wilson loop geometrical center. Then the natural coordinates are $x=(r, h)$, where $r$ and $h$ denote, respectively, the transverse and longitudinal distances to the loop center point.

As far as the structure of QCD string is concerned, theoretically, the effective bosonic string models together with the leading orders in $1 / R$ expansion had been favorable for ages (see, e.g., Ref. [4] and references therein). Their universal common point is that infinitely long QCD string does not exist. Indeed, the string must fluctuate quantum-mechanically and one generically expects [5] that its transverse shape should be Gaussian with logarithmically divergent squared width

$$
\begin{aligned}
\Delta s(h=0, r, R) & =C(R) \exp \left\{-r^{2} / \delta^{2}(R)\right\}, \\
\delta^{2}(R) & =\frac{1}{\pi \sigma} \ln \left(R / R_{0}\right),
\end{aligned}
$$

where $\sigma$ is the string tension and the limit $T \rightarrow \infty$ is understood. On the other hand, the rigorous action sum rules [6] imply that in the leading order in $R$

$$
\delta^{2}(R) \cdot C(R) \propto \sigma
$$

and hence the string is to disappear in the limit $R \rightarrow \infty$. The logarithmic washing out of the string is generic and universal prediction of effective bosonic string models. Note that within this approach only the infrared QCD scale does matter, which is an a priori postulate of the effective models.

If we turn to model-independent considerations, it is crucial that the vacuum expectation $\langle s\rangle_{0}$ is likely to mix up the ultraviolet (lattice spacing $a$ ) and the infrared QCD scales [7]. Namely, up to the powers of $\ln \left(a \Lambda_{Q C D}\right)$ one expects

$$
\langle s\rangle_{0}=\alpha_{0} / a^{4}+\beta_{0} \Lambda_{Q C D}^{2} / a^{2}+\gamma_{0} \Lambda_{Q C D}^{4} .
$$

The first and the last terms are well understood and correspond, respectively, to zero-point fluctuations and the conventional gluon condensate. For the second term there are strong indications [7] 


\begin{tabular}{c|c|c|c|c|c|c|c|c}
\hline$\beta$ & 2.450 & 2.475 & 2.510 & 2.550 & 2.600 & 2.628 & 2.740 & 2.800 \\
\hline$V^{\text {lat }}$ & $24^{4}$ & $26^{4}$ & $32^{4}$ & $34^{4}$ & $40^{4}$ & $36^{4}$ & $36^{4}$ & $40^{4}$ \\
\hline$a, \mathrm{fm}$ & $0.100(1)$ & $0.091(1)$ & $0.081(1)$ & $0.073(1)$ & $0.062(1)$ & $0.056(1)$ & $0.041(1)$ & $0.034(1)$ \\
\hline$V, \mathrm{fm}^{4}$ & $2.4^{4}$ & $2.4^{4}$ & $2.6^{4}$ & $2.5^{4}$ & $2.5^{4}$ & $2.0^{4}$ & $1.5^{4}$ & $1.4^{4}$ \\
\hline
\end{tabular}

Table 1: Simulation parameters.

that it is indeed non-vanishing although is parametrically small. To the best of our knowledge the actual numbers here are not known precisely for $S U(2)$ gauge theory and could be estimated as

$$
\beta_{0} \Lambda_{Q C D}^{2}=[50(10) \mathrm{MeV}]^{2}, \gamma_{0} \Lambda_{Q C D}^{4}=0.02(1) \mathrm{GeV}^{4}
$$

Generically, for the action density $\langle s\rangle_{W}$ within the QCD string an analogous expression holds and then the difference (1) is to be parametrized as

$$
\Delta s=\alpha / a^{4}+\beta \Lambda_{Q C D}^{2} / a^{2}+\gamma \Lambda_{Q C D}^{4},
$$

where we omit $(h, r, R)$-dependencies. While the first term here is expected to vanish, little is known about other contributions. Moreover, since $\Lambda_{Q C D}$ enters both of them, there are no reasons for $\beta$, $\gamma$ coefficients to be identically zero. The prime objective of our paper is to investigate (7) and to present evidences that both IR sensitive terms are indeed non-vanishing (the physical consequences are discussed in concluding remarks).

Our numerical simulations were performed on large set of statistically independent quenched SU(2) configurations generated with standard Wilson action (Table 1). In order to improve the statistics the correlation function (1) was required to be symmetric with respect to $h \rightarrow-h$, thus only even-sized Wilson loops with both temporal and spatial extents up to the half of the lattice size were considered. The signal-to-noise ratio was further enhanced via the standard temporal link integration and APE smearing techniques (see Ref. [1] for comprehensive discussion). The smearing parameters were determined at each $\beta$-value according to [8]; error estimation was done via jackknife method. The action density is symmetric clover-like sum of all neighboring plaquettes contributions. This choice prohibits to consider the points which are too close to the external color sources: the action density operator should not include the links which enter the Wilson loop with integrated temporal edges. Here we differ slightly from the analysis of Ref. [3], where a few exceptional points of this sort had been considered as well. Note, however, that this modification does not change our results and conclusions in any notable way. Furthermore, in the present study we also included additional data set at $\beta=2.80$ with smallest available to us lattice spacing.

Up to this point our setup is similar to that of Ref. [1]. However, the crucial difference is in the methodology of the ground state separation. Technically, the problem is to take the limit $T \rightarrow \infty$, for which the rigorous transfer matrix arguments imply that the leading finite $T$ correction is

$$
\Delta s(h, r, R, T)=\Delta s(h, r, R)+c(h, r, R) \cdot e^{-m(R) T},
$$

where the $h, r, R$ dependence is stated explicitly. Note that the gap $m(R)$ to the next exited state depends upon the quark-antiquark separation $R$ only. This observation allows to improve greatly the quality of $T \rightarrow \infty$ fits. Indeed, it suffices to consider some finite $(h, r)$-range, where signal is 

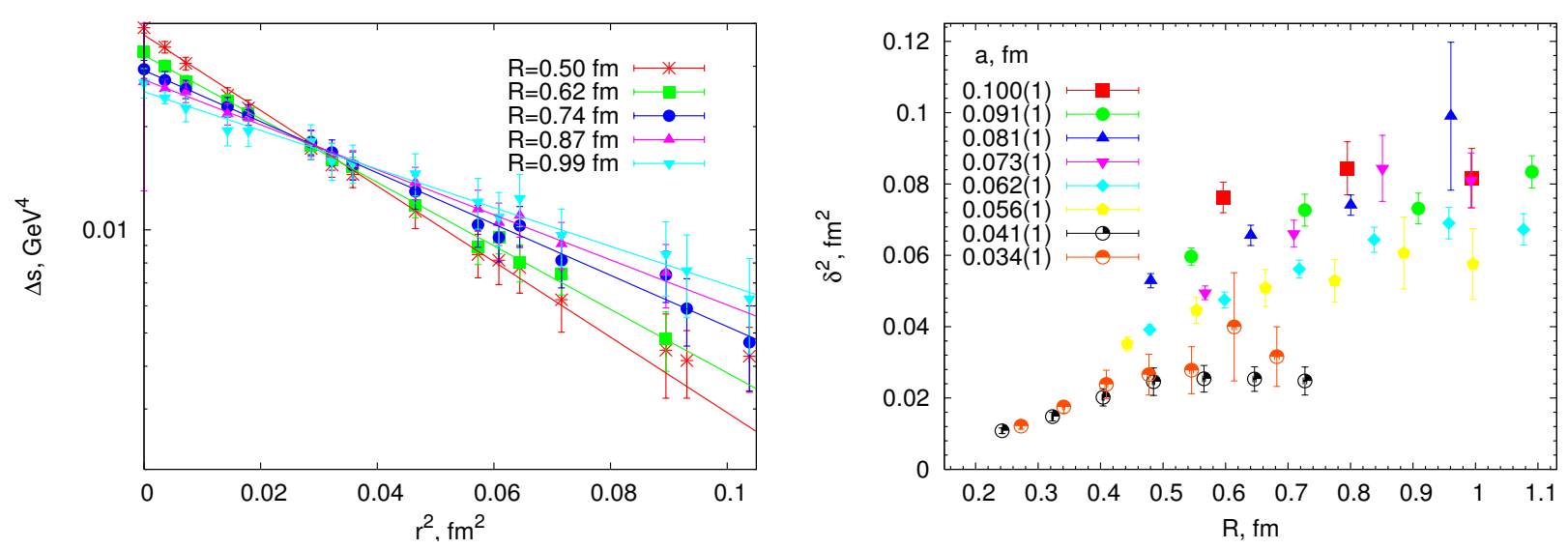

Figure 1: Left: transverse action density profiles taken at $h=0$ and various $R$ on $\beta=2.600$ data set, lines correspond to the best Gaussian fits, Eq. (2). Right: squared string width $\delta^{2}$ versus the string length $R$, graph includes only the points for which both $T \rightarrow \infty$ and Gaussian fits are reliable.

expected to be significant, and fit the data to Eq. (8) with one and the same gap parameter $m(R)$ for all $(h, r)$ points. In practice, the initial $(h, r)$-range was taken to be $h \approx R / 2, r \lesssim[0.3 \mathrm{fm}] / a$, where $0.3 \mathrm{fm}$ is the expected string width scale [1]. Then we varied $(h, r)$ upper limits as well as $T$ fitting range to ensure the fit stability. In most cases the obtained $\Delta s(T=\infty)$ values turned out to be stable with respect to fit parameters variations. Otherwise, the instability of the global fit (8), which happened for several large $R$ data sets, was treated as the lack of statistics, all such points were excluded from further analyzes.

The typical transverse action density profiles obtained at $h=0$ and various $R$ are presented on the left panel of Fig. 1. As a matter of fact, for $R>0.4 \mathrm{fm}$ all our data points (including $r=0$ ) fall indeed onto the Gaussian curve (2) which broadens with rising $R$. Note that the minimal $R$, at which the Gaussian distribution (2) becomes adequate, seems to decrease systematically with diminishing lattice spacing. In particular, for $a<0.05 \mathrm{fm}$ the string theory prediction (2) holds from $R \approx 0.2 \mathrm{fm}$ onwards. The data for squared string width $\delta^{2}(R)$, obtained from the fits to Eq. (2), is presented on Fig. 1 (right panel); only reliable data points, for which both $T \rightarrow \infty$ and Gaussian fits are adequate and stable, are taken into account. It turns out that for $a \geq 0.06 \mathrm{fm}$ the width of the flux tube is essentially lattice spacing independent and is about $\delta \approx 0.3 \mathrm{fm}$ for $1 \mathrm{fm}$ long QCD string. As is apparent from the figure at every fixed lattice spacing the confining flux tube slightly broadens with $R$. Note, however, that the data does not permit us to discuss the logarithmic widening earnestly. The point is not the stability of the logarithmic fits, which one could try out (they are unstable and only allow rough estimation of the relevant parameters). What is crucial is that starting from $a \approx 0.06 \mathrm{fm}$ the string width systematically decreases with diminishing spacing. For instance, at $a \approx 0.04 \mathrm{fm}$ the width of $1 \mathrm{fm}$ long string is of order $0.15 \mathrm{fm}$. Note that this behavior is qualitatively compatible with $\delta \sim a$ dependence, which (if confirmed by other measurements) implies that the string widening observed at finite lattice spacing is a subleading effect which does not survive in the continuum. This rather unexpected conclusion certainly has to be ratified from different viewpoint, to which we turn now.

The shrinking of the QCD string could be assured almost independently via the action sum 

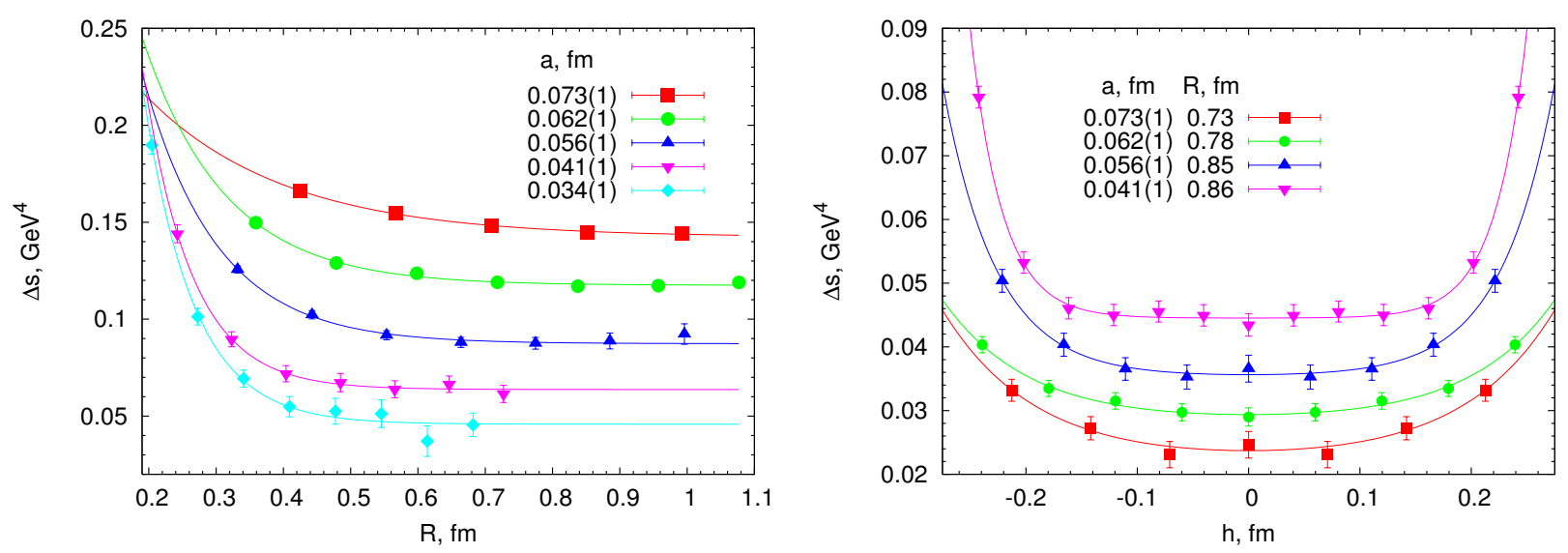

Figure 2: Longitudinal string profiles at $r=0$. Note that on both figures the data points are Y-shifted for readability reasons. Left: $R$-dependence of $\Delta s$ at $h=0$ and various spacings, curves are the best fits to Yukawa-like ansatz (see text). Right: longitudinal string slices at indicated quark-antiquark separations and various spacings. Curves represent the best fits to Eq. (9).

rules (4) once the Gaussian shape of the transverse profile had been established for $r \geq 0$. To this end let us focus on the action density difference (1) at the string geometrical center $r=h=0$, at which $\Delta s$ is a function of $R$ and $a$ only. The problem here is the reliable extrapolation to the limit of large quark-antiquark separations, for which theoretically grounded ansatz like (8) does not exist. Our solution is purely experimental: we observe that at every fixed spacing the $R$-dependence of the action density at the string central point is well described by simple Yukawa-like ansatz $\Delta s(R)=\Delta s+$ const $\cdot \exp (-M R / 2)$, see the left panel of Fig. 2 . Then the quality of fits could be greatly improved by considering simultaneously all available $h$-points at $r=0$. Indeed, for $h \neq 0$ the above Yukawa ansatz generalizes to

$$
\Delta s(h, R)=\Delta s+A \cdot e^{-M R / 2} \cdot \cosh (M h) .
$$

Despite of its simplicity Eq. (9) works surprisingly well and correctly describes all available numerical data (see Fig. 2 for illustration). In turn, the fits suggest strongly that both parameters $A(a)$, $M(a)$ are divergent in continuum with leading divergences being compatible with $A \sim 1 / a^{4}$ and $M \sim 1 /\left(a^{2} \Lambda_{Q C D}\right)$. Taken at face value the data indicates that in the continuum limit the confining string appears immediately once the quark pair is created and its longitudinal profile has no sign of Coulomb-like tails coming from color sources (excluding, of course, the positions of quarks, where self-energy singularities are generically expected). Note, however, that this doesn't deny a Coulomb piece in the static potential, which is present indeed and scales with $a$ in usual way, see below.

The action density difference at the string geometrical center in the limit of large quarkantiquark separation as is determined via Eq. (9) is shown on the left panel of Fig. 3. As is apparent from that figure, the data points suggest that $a^{2} \cdot \Delta s$ is linear in $a^{2}$ and has non-zero intercept with the vertical axis. Thus we conclude that the difference between the action density at the QCD string geometrical center and its vacuum expectation value is likely to diverge quadratically in the 

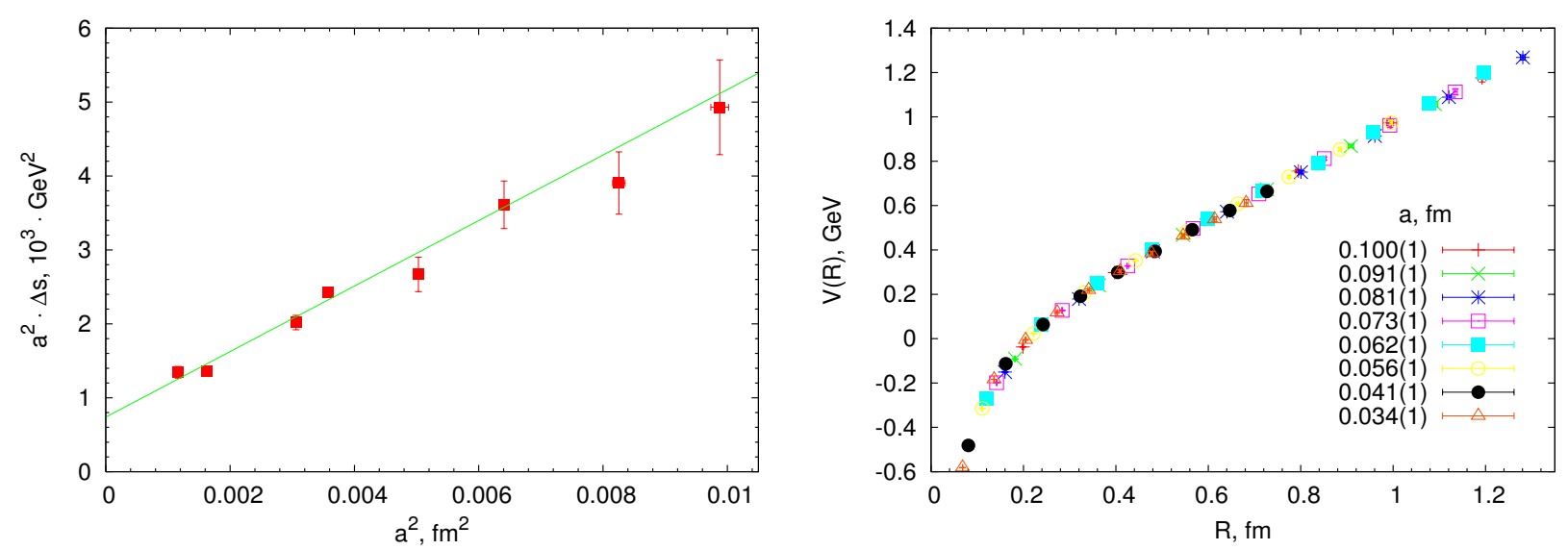

Figure 3: Left: lattice spacing dependence of the action density at the string geometrical center in the limit of large quark-antiquark separation. Right: scaling of the heavy quark potential with lattice spacing. Note that the constant contribution had been subtracted.

continuum limit

$$
\begin{gathered}
\Delta s=\frac{\beta \Lambda_{Q C D}^{2}}{a^{2}}+\gamma \Lambda_{Q C D}^{4} . \\
\beta \Lambda_{Q C D}^{2}=[27(2) \mathrm{MeV}]^{2}, \gamma \Lambda_{Q C D}^{4}=0.018(2) \mathrm{GeV}^{4} .
\end{gathered}
$$

Few comments are now in order:

i) The last term in Eq. (10) corresponds to the conventional gluon condensate and the numerical value quoted in (11) is fairly compatible with its best known to us estimates in $S U$ (2) gluodynamics (cf. Eq. (6)). Thus we conclude that the gluon condensate vanishes on the symmetry axis of long QCD string.

ii) At first sight, the quadratically divergent term looks suspicious since its numerical value is rather unusual for the quenched theory. However, this term is known to be small, see Eq. (6). Moreover, we definitely exclude the possibility that it is caused by the finite volume effects. Indeed, all our data points fall practically onto the same curve, however, the corresponding physical volumes differ significantly. On the other hand, it is the parametric smallness of the divergent term which explains the observed constancy of the string width for $a \gtrsim 0.06 \mathrm{fm}$. Essentially at this lattice spacing the eventually divergent term becomes comparable with the gluon condensate and starts to dominate the action density, squeezing the flux tube.

iii) Finally, we note that despite of the seemingly vanishing string width and the divergent action density, the rough estimation of the product $\delta^{2} \cdot C$ reveals that it has no sign of power-like lattice spacing dependence as is required by Eq. (4). Moreover, the heavy quark potential scales correctly, see Fig. 3 (right). Hopefully, the same holds for other physical observables as well.

To summarize: the central point of our paper is to argue that in the Yang-Mills theory dependence upon the UV scale is likely to be much more intricate than it is usually thought to be. Our results constitute an additional confirmation of this, however, the prime message encoded in the paper is not new. Indeed, there are accumulating evidences that a kind of UV/IR mixing, or fine tuning, is inherent to Yang-Mills theory. Historically, it was discussed first in connection with center vortices [9], which tuned out to be infinitely thin surfaces with nonetheless physical total 
area. Then the analogous phenomenon was discovered in the vacuum topological density: it had been shown [10] that topology is concentrated within the percolating three-dimensional domains possessing divergent topological density (for further examples and thorough discussion see, e.g., Refs. $[7,11]$ and references therein). What is in common for both these examples, is that neither the center vortex thickness nor the vacuum topological density are directly observable. In this respect our results are indeed remarkable, since it became a common wisdom to believe that the string theory prediction $(2,3)$ undoubtedly holds for QCD string. However, we were not able to imaging a physically meaningful experiment which would assert the validity of either (2) or (3) separately. It seems that only their "integral version", Eq. (4), is meaningful and indeed no sign of lattice spacing dependence had been seen in the heavy quark potential. Therefore, our findings are similar to the above example, for which the observable integral effect is encoded into the topological susceptibility. The only essential difference is that the object, for which the fine tuning was discussed, is rather unexpected in this context.

The authors are grateful to the members of ITEP Lattice Group for stimulating environment. The thorough discussions with V.I. Zakharov, V.I. Shevchenko and E.T. Akhmedov are kindly acknowledged. The work was partially supported by the grants RFBR-05-02-16306a, RFBR-07-02-00237a. F.V.G. and S.M.M. were also supported by INTAS YS grants 04-83-3943 and $05-109-4821$.

\section{References}

[1] G. S. Bali et al., Phys. Rev. D 51, 5165 (1995).

[2] R. W. Haymaker et al., Phys. Rev. D 53, 389 (1996); F. Okiharu, R. M. Woloshyn, Nucl. Phys. Proc. Suppl. 129, 745 (2004); A. M. Green et al., Phys. Rev. D 55, 1216 (1997); F. Bissey et al., Nucl. Phys. Proc. Suppl. 141, 22 (2005), hep-lat/0606016.

[3] P. Y. Boyko, F. V. Gubarev and S. M. Morozov, arXiv:0704.1203 [hep-lat].

[4] M. Luscher, P. Weisz, JHEP 0207, 049 (2002); JHEP 0407, 014 (2004).

[5] M. Luscher et al., Nucl. Phys. B 180, 1 (1981).

[6] F. Karsch, Nucl. Phys. B 205, 285 (1982); C. Michael, Nucl. Phys. B 280, 13 (1987); H. J. Rothe, Phys. Lett. B 355, 260 (1995).

[7] G. Burgio et al., Phys. Lett. B 422, 219 (1998); R. Akhoury, V. I. Zakharov, Phys. Lett. B 438, 165 (1998); G. S. Bali, Phys. Lett. B 460, 170 (1999); V. I. Zakharov, Nucl. Phys. Proc. Suppl. 164, 240 (2007), hep-ph/0309178; F. V. Gubarev et al., Surveys High Energ. Phys. 15, 89 (2000);

P. E. L. Rakow, PoS LAT2005, 284 (2006); P. Y. Boyko et al., Phys. Rev. D 73, 014512 (2006).

[8] C. Legeland et al., Nucl. Phys. Proc. Suppl. 63, 260 (1998).

[9] F. V. Gubarev et al., Phys. Lett. B 574, 136 (2003).

[10] I. Horvath et al., Phys. Rev. D 68, 114505 (2003); C. Aubin et al., Nucl. Phys. Proc. Suppl. 140, 626 (2005); F. V. Gubarev et al., JETP Lett. 82 (2005) 343; P. Y. Boyko, F. V. Gubarev, Phys. Rev. D 73, 114506 (2006); Y. Koma et al., PoS LAT2005, 300 (2006).

[11] V. I. Zakharov, Phys. Atom. Nucl. 68, 573 (2005); AIP Conf. Proc. 756, 182 (2005); hep-ph/0612341; hep-ph/0602141; Nucl. Phys. Proc. Suppl. 164, 240 (2007). 